\title{
Interactive comment on "Optimal use of buffer volumes for the measurement of atmospheric gas concentration in multi-point systems" by A. Cescatti et al.
}

\section{Anonymous Referee \#2}

Received and published: 8 June 2016

General remarks: The study by Cescatti et al. investigates the uncertainty reduction of a multi-point $\mathrm{CO} 2$ concentration measurement setup using a single gas analyser due to optimal buffer volumes. The results can support ongoing activities within the ICOS ecosystem network to optimize GHG concentration profile measurement setups to correctly record the GHG storage change. Besides the functions and dependencies presented in the manuscript I would only recommend to show a dependency of the optimal buffer volume on the flow and the number of measurement points. The manuscript includes clear, concise and comprehensible explanations of the approach and the results. It is appropriate for AMT and I recommend the acceptance of the manuscript after consideration of my very few remarks. 
Minor remarks P3L4: "closed-path" instead of "close-path" P3L8: please indicate the type of the ultrasonic anemometer (HS?, R3?)

Interactive comment on Atmos. Meas. Tech. Discuss., doi:10.5194/amt-2016-18, 2016.

Interactive comment 